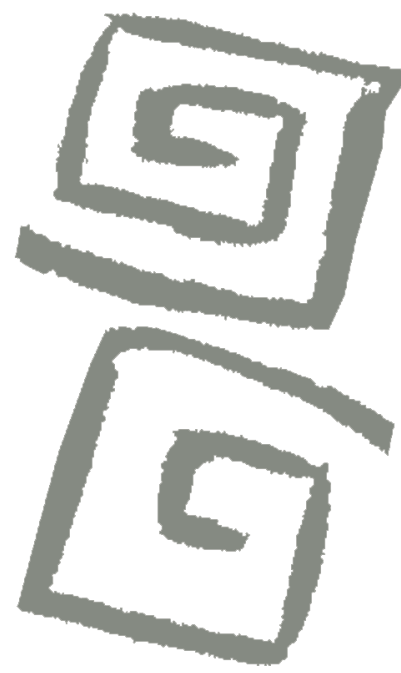

\title{
Dificultades del autocuidado masculino: discursos de hombres participantes en un grupo de educación para la salud
}

\author{
Barriers to self-care among men: discourses of men \\ participating in a health education group
}

Francisco Timbó de Paiva Neto ${ }^{1}$, Paula Fabrício Sandreschi ${ }^{2}$, Maria Socorro de Araújo Dias', Mathias Roberto Loch ${ }^{4}$

${ }^{1}$ Magíster en Educación Física. Estudiante de Doctorado, Universidade Federal de Santa Catarina. Florianópolis, Santa Catarina, Brasil. $\bowtie$ (iD)

${ }^{2}$ Magíster en Ciencias del Movimiento Humano. Estudiante de doctorado, Universidade Federal de Santa Catarina. Florianópolis, Santa Catarina, Brasil. $\square$ (iD)

${ }^{3}$ Doctora en Enfermería. Profesora, Universidade Estadual Vale do Acaraú. Sobral, Ceará, Brasil. $\bowtie$ iiD

${ }^{4}$ Doctor en Salud Colectiva. Profesor Universidade Estadual de Londrina. Londrina, Paraná, Brasil. $ه$ (iD)
RESUMEN El objetivo de este estudio fue analizar las dificultades de la participación masculina en los servicios de salud y sus prácticas de autocuidado en el proceso saludenfermedad. Para ello, se realizó una intervención, a la luz de la educación en salud, con hombres que vivían en el área de cobertura de una unidad de atención primaria, en un municipio del nordeste de Brasil. La intervención fue diseñada por profesionales de la unidad y la tercera etapa consistió en cinco encuentros grupales que abordaron temas relacionados con la salud masculina. Para el análisis de los discursos se utilizó la estrategia de análisis de contenido. La experiencia permitió reflexionar sobre la importancia del desarrollo de acciones estratégicas con el propósito de promover el acercamiento del público masculino al servicio de salud y el autocuidado de los usuarios, especialmente en el escenario de atención primaria, en el marco de la Política Nacional de Atención Integral de Salud para el Hombre (PNAISH). Resignificar las acciones que involucran a la salud del hombre y el cambio de actitud profesional para atender esta población puede desencadenar en el público masculino la sensación de pertenencia al espacio de promoción, protección y recuperación de la salud.

PALABRAS CLAVES Género; Salud del Hombre; Atención Primaria de Salud; Promoción de la Salud; Educación en Salud; Brasil.

ABSTRACT The objective of this study was to analyze men's discourses regarding difficulties related to their participation in health services and their self-care practices in the health-disease process. In order to do so, a health education initiative was implemented with men living in the area covered by a Basic Health Unit (UBS) in a municipality in Northeastern Brazil. The initiative was designed by professionals at the UBS, and its third stage consisted of five group meetings that addressed issues related to men's health. For the analysis of discourses, a content analysis strategy was used. The experience allowed us to reflect on the importance of developing strategic actions oriented towards promoting men's engagement with health services and the self-care of users, particularly in primary care settings. This is considered within the framework of the National Policy for Comprehensive Men's Healthcare (PNAISH). Re-signifying actions oriented towards men's health and changing the perspectives of professionals attending to this population can create a sense of belonging in men with respect to spaces of health promotion, protection, and recovery. KEY WORDS Gender; Men's Health; Primary Health Care; Health Promotion; Health Education; Brazil. 


\section{INTRODUCCIÓN}

Las evidencias indican que la participación de hombres, en todos los niveles de atención de la salud, es muy inferior a la de las mujeres ${ }^{(1)}$, incluso en actividades de promoción de la salud, como grupos de prácticas saludables y de actividad física ofrecidos en los escenarios de atención primaria. Otro factor relacionado con esta realidad es que la mayor demanda de atención por parte de la población masculina se da solo en el marco de enfermedades agudas, o sea, la demanda se da principalmente a partir de dolencias agudas $^{(2)}$. Otros factores relacionados con la ausencia masculina en los servicios de atención primaria de la salud también son la presencia mayoritaria de profesionales mujeres, cuestiones culturales ${ }^{(2)}$, además de la falta de estímulo por parte de los equipos de salud con acciones y programas dirigidos específicamente a los hombres ${ }^{(3)}$. Esta situación incluso provoca que muchas veces las enfermedades se detecten en estadios avanzados, fragilizando el proceso de recuperación y aumentando la posibilidad de fallecimiento ${ }^{(4)}$.

Considerando ainda que la construcción de la masculinidad predominante en Brasil no estimula a los hombres al cuidado de sí o del otro ${ }^{(5,6)}$ el Ministerio de Salud, intentando enfrentar esta situación, creó, en 2008, la Política Nacional de Atención Integral a la Salud del Hombre (PNAISH), que tiene como uno de los objetivos promover acciones de salud que contribuyan a comprender la singularidad masculina en los contextos socioculturales y político-económicos, buscando aumentar la expectativa de vida y la reducción de los índices de morbimortalidad por causas prevenibles y evitables en esta población ${ }^{(4)}$.

En vista de cumplir los objetivos propuestos por la PNAISH, ciertos programas y acciones ofrecidos a la comunidad buscan atender necesidades específicas de este público ${ }^{(7)}$. Una estrategia que ha sido implementada es la inclusión de acciones de educación en salud en los espacios de atención primaria, las que engloban tres segmentos: los profesionales de la salud con relación a la prevención de dolencias y la promoción de la salud; los gestores que apoyan a esos profesionales; y la población que necesita construir conocimientos y aumentar su autonomía en los cuidados individuales y colectivos $^{(8)}$. De esta forma, para atender a los hombres, brindarles la atención que necesitan y considerar sus particularidades, la educación en salud puede ser potente en el sentido de favorecer la aproximación de estos con los servicios de salud, sobre todo de atención primaria.

La educación en salud como proceso político-pedagógico requiere del desarrollo de un pensamiento crítico y reflexivo acerca del proceso salud-enfermedad, que permita develar la realidad y proponer acciones transformadoras, en tanto sujeto histórico y social capaz de operar y opinar sobre las decisiones de salud para el cuidado de sí, de su familia y de la colectividad ${ }^{(9)}$. Para algunos educadores, la educación permanente en salud surge de la educación popular, sobre la base de ciertos principios y/o directrices elaborados por Paulo Freire como, por ejemplo, la educación como práctica de la libertad y la búsqueda del aprendizaje significativo ${ }^{(10)}$.

Considerando que la atención primaria se presenta como puerta de entrada de los servicios de salud en Brasil, y que las acciones desarrolladas deben ir más allá de la asistencia curativa, englobando también acciones preventivas y de promoción de la salud, enfocándose incluso en la población masculina, el objetivo de este artículo fue analizar el discurso masculino respecto de las dificultades para el autocuidado por medio de las relatos de participantes hombres de un grupo de educación en salud, centrado en la salud masculina.

\section{MÉTODOS}

Se trata de una investigación de intervención, de carácter cualitativo del tipo descriptivoexploratoria, realizada entre agosto de 2016 y enero de 2017, en una unidad de atención primaria, ubicada en el municipio de Sobral, Ceará, en la región nordeste de Brasil. 
La unidad de atención primaria en la que realizó la intervención contaba con dos equipos de salud familiar, un equipo de salud bucal y un equipo del Núcleo de Apoyo a la Salud Familiar. Según datos del último Semanario de Territorialización del Municipio de Sobral, realizado en 2016, la unidad de atención primaria en cuestión contaba con 1.711 familias registradas, y un total de 5.902 personas.

La intervención se realizó en cuatro etapas: 1$)$ presentación a los profesionales de la unidad de atención primaria; 2) análisis de la visión de la comunidad sobre la participación masculina en los espacios de salud; 3) conformación del grupo sobre salud masculina; 4) repercusiones de la intervención. La Figura 1 muestra las etapas de la intervención y sus respectivos procedimientos y la Figura 2 presenta el modelo lógico de la intervención, con la relación entre objetivos, insumos, actividades, productos, resultados y factores influyentes. Para este estudio, se consideraron solo los relatos de los hombres de la comunidad que participaron de la etapa 3 de la intervención, relacionada con aspectos culturales de masculinidades y autocuidado masculino.

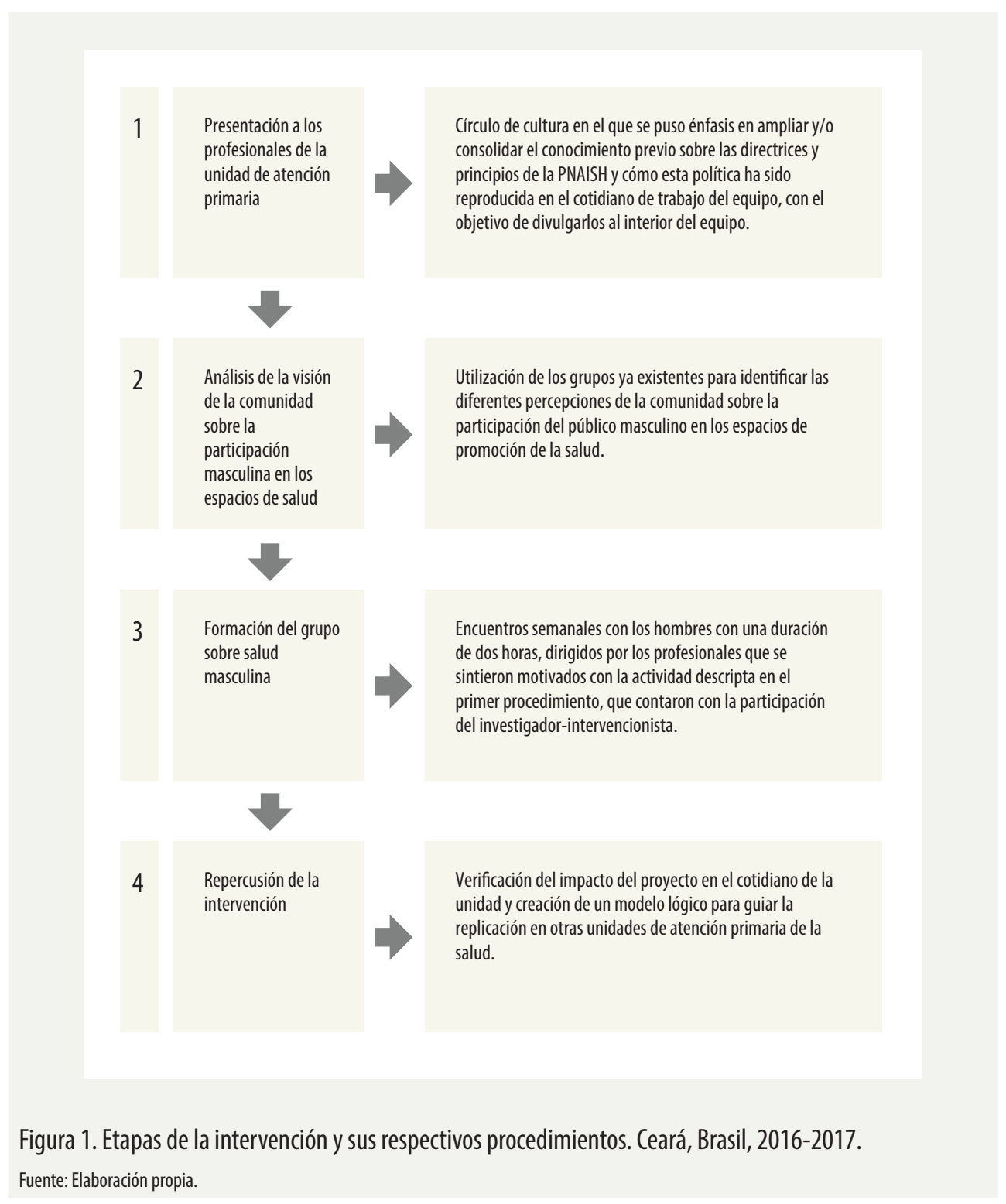


OBJETIVO GENERAL

Propiciar la participación de la población masculina en los ambientes de promoción de la salud a partir de la construcción de un espacio de encuentros sistemáticos en la Estrategia de Salud Familiar (ESF) del municipio de Sobral, estado de Ceará, Brasil.

OBJETIVOS ESPECÍFICOS

Comprender cómo la

PNAISH se incorpora a

la atención primaria de

la salud.

Verificar la visión de la

comunidad sobre la

temática.

Desarrollar de forma

colaborativa una

agenda sistemática de

acciones promotoras

de la salud del

hombre.
INSUMOS

Profesionales de la
atención primaria:
agentes de salud, personal
de enfermería, dentistas,
auxiliares de salud bucal,
técnicos de enfermería,
personal de farmacia,
auxiliares administrativos,
auxiliares de servicios
generales, médicos,
gerentes, jefes de
residencia, residentes.
Hombres de la comunidad.
Comunidad en general.

ACTIVIDADES

Identificación de la
percepción de la
comunidad sobre la
promoción de la salud
masculina.
Encuentros y acciones
en educación en salud
masculina.
Análisis de las acciones
implementadas en la
visión de los
profesionales de la
salud de las unidades
de atención primaria.

PRODUCTOS

Trabajos científicos y
monografías de
finalización de cursos
de Residencia
Multiprofesional en
Salud Familiar
Presentación de los
resultados de los
encuentros en la
reunión de la unidad
de atención primaria,
como forma de
educación permanente
para los profesionales.

RESULTADOS

Institucionalización del programa.

Acercamiento entre $\mathrm{e}$ equipo de salud y los usuarios.

Ampliación de la participación masculina y apertura del espacio hacia sus percepciones en un ambiente de promoción de la salud.

\section{FACTORES INFLUYENTES}

Negativos: horario de funcionamiento del centro de salud, mayoría de profesionales mujeres, factores socioculturales, otras prioridades en el contexto masculino (empleo, familia, etc.), ausencia de programas y atención específica para hombres en el contexto de la atención primaria de la salud, desfamiliarización de los profesionales con la atención del público masculino.

Positivos: compromiso de los profesionales de la salud, encuentros en horarios favorables, encuentros coordinados por profesionales hombres, apoyo de la Secretaría Municipal de Salud, colaboración y compromiso de la comunidad, espacio físico adecuado para la realización del proyecto.

Figura 2. Modelo lógico de intervención: relación entre objetivos, insumos, actividades, productos, resultados y factores influyentes. Fuente: Elaboración propia.

La intervención tuvo una duración total de seis meses, de los cuales tres meses se destinaron a los encuentros del grupo de educación en salud centrado en el autocuidado masculino, realizados en el auditorio de la unidad de atención primaria, cada dos semanas, lo miércoles de $18 \mathrm{~h}$ a 19:30 h. Para el primer encuentro, la elección de un horario posterior al cierre del horario comercial, buscó incentivar una mayor participación de los trabajadores, y fue acatada por los participantes, manteniéndose hasta el final de la intervención. Cabe destacar que solo el primer encuentro del grupo tuvo un tema sugerido por los profesionales, mientras que los demás temas fueron elegidos por los hombres participantes del primer encuentro. Cada encuentro llevaba un título, extraído de canciones interpretadas por artistas hombres de la música popular brasileña, cuya letra permitía reflexionar sobre cuestiones relacionadas con la masculinidad. El investigador a cargo de la intervención consideró que las canciones elegidas para iniciar cada grupo servían como estrategia disparadora de discusión en el grupo de hombres.

En la Tabla 1 se presentan el orden, el título, la temática abordada relacionada con la salud y el número de participantes de cada uno de los encuentros.

Para participar del grupo de hombres, los sujetos debían ser usuarios, del sexo masculino, y residir en la zona de alcance de la unidad de atención primaria elegida. Todos los 
Tabla 1. Orden, título, temática abordada relacionada con la salud y número de participantes en cada encuentro del estudio. Sobral, Ceará, 2016-2017.

\begin{tabular}{|c|c|c|c|c|}
\hline Orden & Título del encuentro & Título de canción y autores & Temática abordada & Número de participantes \\
\hline $1^{0}$ & $\begin{array}{l}\text { "Viví con la ilusión de que ser } \\
\text { hombre bastaría" }\end{array}$ & $\begin{array}{l}\text { Super-Homem, A canção } \\
\text { (Gilberto Gil) }\end{array}$ & $\begin{array}{l}\text { Prevención del cáncer de } \\
\text { próstata }\end{array}$ & 21 \\
\hline $2^{0}$ & $\begin{array}{l}\text { "Hombre no llora ni por dolor } \\
\text { ni por amor" }\end{array}$ & $\begin{array}{l}\text { Homem não chora (Alvin L / } \\
\text { Frejat) }\end{array}$ & $\begin{array}{l}\text { Relación con el servicio de } \\
\text { salud }\end{array}$ & 20 \\
\hline $3^{0}$ & "Ese tipo soy yo" & $\begin{array}{l}\text { Esse cara sou eu (Roberto } \\
\text { Carlos) }\end{array}$ & $\begin{array}{l}\text { Prevención de enfermedades } \\
\text { cardiovasculares }\end{array}$ & 18 \\
\hline $4^{0}$ & $\begin{array}{l}\text { "Ser un hombre femenino no } \\
\text { afecta mi lado masculino" }\end{array}$ & $\begin{array}{l}\text { Masculino e feminino (Baby } \\
\text { Consuelo / Didi Gomes / Pepeu } \\
\text { Gomes) }\end{array}$ & $\begin{array}{l}\text { Lepra: cómo identificarla y } \\
\text { cómo cuidarse }\end{array}$ & 19 \\
\hline $5^{0}$ & "SSoy hombre con Hy como soy!" & Homem com H (Antônio Barros) & Salud bucal & 20 \\
\hline
\end{tabular}

hombres que participaron de la intervención recibieron una invitación impresa en su residencia, que fue entregada por los agentes comunitarios de salud o por algún familiar que se presentaba con frecuencia en la unidad de atención primaria. La invitación fue redactada por los profesionales de la salud y contenía información sobre el objetivo del grupo, fecha y horario del encuentro inicial. Por cuestiones estructurales, de espacio físico y de preservar la calidad de los sentidos producidos con la intervención, se consideró abrir solo 20 vacantes para los hombres de la comunidad.

Los datos se obtuvieron con el método "círculo de cultura", propuesto por Monteiro y Vieira ${ }^{(11)}$, con base en la pedagogía de Paulo Freire. El círculo de cultura se utilizó como herramienta de manejo de los espacios y como estrategia para la colecta de datos en lo que se refiere a provocar el inicio de los relatos de los participantes. Esta metodología integra los principios del cuidado con enfoque en la promoción de la salud y busca brindar instrumentos a los profesionales para desarrollar acciones educativas en salud y proveer autonomía al usuario en su proceso de salud-enfermedad. Los relatos de los participantes del grupo se grabaron en audio para asegurar una transcripción y análisis fidedigno del material obtenido.

Luego de la recolección de datos, se analizaron los relatos con base en el método de análisis de contenido, utilizando la técnica de análisis temático, en la cual el texto se separa en unidades de significación ${ }^{(12)}$. Operacionalmente, el análisis de los discursos se dividió en tres etapas, que se detallan a continuación.

1) Preanálisis: luego de la colecta de datos, se escucharon los relatos, en promedio, dos o tres veces, para posibilitar la transcripción integral. A continuación, se eligieron los documentos a ser analizados, permitiendo retomar los objetivos de la investigación. Finalmente, se elaboraron los indicadores que orientaron la interpretación final del material analizado.

2) Exploración del material: en esta etapa se realizó la significación. Primero, en los textos transcritos se recortaron las unidades de registro: una palabra, una frase o un acontecimiento.

3) Clasificación de datos: se agruparon los datos en las categorías teóricas o empíricas que orientaron la especificación de los temas. Luego, esas categorías se agruparon de acuerdo con sus significados, dando origen a dos categorías de significación: aspectos culturales de la invisibilidad masculina y promoción del autocuidado masculino.

El estudio fue evaluado por el Comité de Ética en Pesquisa con Seres Humanos de la Universidade Estadual Vale del Acaraú (UVA), y aprobado a través del protocolo $\mathrm{N}^{\circ} \mathrm{CAAE}$ 62963316.9.0000.5053. Todos los participantes firmaron el consentimiento libre e 
informado, de acuerdo a lo establecido en la Resolución 196/96 del Consejo Nacional de Salud, y se garantizó el anonimato y la confidencialidad de los datos. Para evitar la identificación de los autores de los relatos, los nombres de los participantes fueron sustituidos por letras.

\section{RESULTADOS}

En los cinco encuentros realizados, el número mínimo de hombres participantes fue de 18 y el máximo de 21. Considerando la característica de los 21 participantes, la media de edad era de 44 años (desvío estándar $=15,7$ años). La mayoría de los participantes tenía entre cinco y nueve años de estudio $(71,4 \%)$, no tenía compañero o compañera $(57,2 \%)$, mencionó tener una o dos enfermedades $(42,8 \%)$, presentaba exceso de peso $(61,9 \%)$ y residía hacía más de 10 años en el barrio $(71,4 \%)$. Las características sociodemográficas relacionadas con las condiciones de salud de los participantes del grupo se detallan en la Tabla 2.

Algunos relatos muestran el estereotipo del "hombre fuerte", de la enfermedad como señal de debilidad (aunque en el relato que presenta esta última idea, se asocie este tipo de visión a algo del pasado) y a la falta de "costumbre" de cuidarse:

¡El hombre es muy bravo! Yo digo que el hombre es como un perro cazador... Trabaja todo el día y no le da importancia a cuidarse, y ahí se termina muriendo joven. (Hombre B)

Antiguamente decían que el hombre que se enferma mucho es débil y no sirve para casarse. (Hombre D)

Ah, sabes cómo es ¿no? No crecimos con la costumbre de cuidarnos. (Hombre C)

Uno de los relatos hace alusión a que el principal determinante de esta situación es cultural/educacional, atribuyendo a los padres
Tabla 2. Características sociodemográficas y condiciones de salud de los participantes del estudio. Sobral, Ceará, 2016-2017.

\begin{tabular}{|c|c|c|}
\hline \multirow{2}{*}{ Variables } & \multicolumn{2}{|c|}{ Grupo de hombres $(n=21)$} \\
\hline & $\mathrm{n}$ & $\%$ \\
\hline \multicolumn{3}{|l|}{ Franja etaria } \\
\hline 30 años o menos & 6 & 28,5 \\
\hline 31 a 59 años & 10 & 47,7 \\
\hline 60 años o más & 5 & 23,8 \\
\hline \multicolumn{3}{|l|}{ Escolaridad } \\
\hline $0-4$ & 3 & 14,3 \\
\hline $5-9$ & 15 & 71,4 \\
\hline 10 o más & 3 & 14,3 \\
\hline \multicolumn{3}{|l|}{ Estado civil } \\
\hline Con compañero/a & 9 & 42,8 \\
\hline Sin compañero/a & 12 & 57,2 \\
\hline \multicolumn{3}{|c|}{ Morbilidades (número) } \\
\hline 0 & 7 & 33,4 \\
\hline 102 & 9 & 42,8 \\
\hline 30 más & 5 & 23,8 \\
\hline \multicolumn{3}{|l|}{ Exceso de peso ${ }^{a}$} \\
\hline No & 8 & 38,1 \\
\hline Sí & 13 & 61,9 \\
\hline \multicolumn{3}{|c|}{ Tiempo de residencia (años) } \\
\hline $0 \mathrm{a} 4$ & 2 & 9,5 \\
\hline 5 a 9 & 4 & 19,1 \\
\hline 10 o más & 15 & 71,4 \\
\hline
\end{tabular}

(del sexo masculino) al menos parte de la responsabilidad:

Se pasa mucho de padre a hijo, que el hombre no puede llorar, no puede enfermarse, no puede hablar sobre lo que está sintiendo y eso va fijándose en nuestra cabeza. (Hombre L)

Algunos relatos señalaron la cuestión de la necesidad de superación del estereotipo que dificulta el autocuidado:

Necesitamos poner mucha atención en la salud, iy yo lo hago! ¡Pero no dejo de ser hombre por eso! (Hombre E) 
Nosotros también necesitamos cuidarnos. Somos seres humanos y también tenemos nuestras debilidades. (Hombre A)

Es interesante acercarse al centro de salud sin estar enfermo. El hombre también tiene que cuidarse. (Hombre F)

Uno de los aspectos más relevantes de este trabajo está relacionado a los diversos motivos señalados por los hombres participantes como aspectos que dificultan la adopción del autocuidado. Los relatos mencionan la cuestión de la falta de tiempo, el desconocimiento sobre los servicios prestados por los servicios de salud e incluso la vergüenza de hablar sobre el asunto, además de factores como, por ejemplo, que los servicios de salud estén integrados principalmente por mujeres, que estos servicios no tienen una visión ampliada sobre la salud del hombre, el horario de funcionamiento de los servicios de atención primaria e, incluso, en la "gastada" de los colegas del trabajo:

Decimos que no tenemos tiempo para venir al grupo, pero cuando sintamos algo vamos a tener que encontrar el tiempo para ir al médico. (Hombre E)

No sabía que el centro de salud tenía este tipo de actividad para conversar y aconsejarnos. Para mí, la charla acá es muy rica y hay otros grupos. (Hombre B)

Siempre que necesitaba atenderme iba derecho al hospital, pero conversando acá con el doctor vi que se pueden resolver muchas cosas en el centro, e incluso es más cerca de casa. (Hombre N)

En la primera reunión preguntaron si conocía la próstata, dije que sí, pero no sabía ni lo que era, me sentí desinformado. Al comienzo todos se reían con vergüenza al hablar sobre el tema, pero al final todo el mundo aprendió sobre algo que es importante para nosotros los hombres. (Hombre F)
Como en el centro de salud la mayoría de los profesionales son mujeres, no sé, parece que están más acostumbradas a atender a mujeres y criaturas. (Hombre M)

El personal de salud piensa que el hombre es solo una próstata. Hay otras cosas que hay que cuidarse, están las enfermedades, los tratamientos y todo, pero solo ven al hombre en el centro de salud en noviembre. (Hombre B)

El horario del centro de salud tampoco ayuda, solo acá en el grupo me entero de lo que sucede, pero es porque solo puedo venir a la noche. (Hombre $\mathrm{H}$ )

En el trabajo me cargan cuando digo que fui al médico. No me engancho, me estoy cuidando. (Hombre C)

\section{DISCUSIÓN}

Entre los relatos analizados de los hombres participantes de la intervención, algunos puntos merecen ser destacados. Entre ellos, el reconocimiento del estereotipo del hombre "fuerte" que se origina, sobre todo, en la "transmisión de padre a hijo" y, al mismo tiempo, el reconocimiento de la necesidad de superar ese estereotipo. Entre los posibles aspectos que dificultan la superación de la invisibilidad en los servicios, los hombres citaron factores importantes, como ciertas características de los hombres (que a veces se sienten avergonzados con algunas situaciones) $y$, principalmente, aspectos relacionados con los propios servicios de salud, entre los cuales están: el horario de funcionamiento de los servicios de salud, la minoría de hombres en el plantel de trabajadores de atención primaria y la ausencia de una visión más amplia sobre la salud del hombre por parte de estos servicios). Además, vale destacar la falta de conocimiento, por parte del público masculino, del alcance de los servicios de atención primaria, que de algún modo señala, por un lado, la necesidad de una mayor divulgación por parte 
de los servicios y, por otro, la presión que otras personas pueden ejercer con relación al hombre que busca autocuidado de calidad.

Los resultados muestran un panorama de nuevas posibilidades con relación a la percepción del hombre inserto en el sistema de salud, al significado que le atribuye a la masculinidad y a los cambios en su proceso de autocuidado. El abordaje enfocado en el hombre, puede resignificar tanto su percepción sobre la masculinidad como su rol en cuanto al género masculino, y cuidarse a partir de la experiencia de la participación del grupo de educación en salud.

Esos roles se determinan en función de las necesidades y de los valores dominantes de una sociedad ${ }^{(5,13)}$. El imaginario tradicional del hombre fuerte e indestructible refuerza dos puntos del modelo de masculinidad hegemónico. Si, por un lado, la fuerza y la salud reafirman la virilidad del hombre, por otro, es la capacidad de sustentarlos y mantenerlos que consolida la fuerza moral masculina ${ }^{(14)}$.

Por lo tanto, aunque su identidad cultural no se relaciona con el cuidado, al perder el rol de inquebrantable, el hombre vio cómo su imagen de fuerte y viril fue progresivamente eclipsada ${ }^{(15)}$. Ante ese escenario, su la participación en los servicios de salud fomenta la reflexión sobre la masculinidad y la consolidación de relaciones más democráticas e igualitarias en la esfera de la salud ${ }^{(16,17)}$. Como relató uno de los participantes, esa nueva construcción permite la coexistencia de una convivencia más afectiva entre el ser hombre frágil y destructible y el ideal de lo que es "ser un hombre de verdad". Esa nueva imagen fue posible debido a la resignificación de la propia masculinidad, percibida en los relatos de los participantes.

Así, cabe recordar que los participantes residen en una región del país marcada por el estigma, la violencia y la influencia machista $^{(18,19)}$. En la construcción de las identidades masculinas, esa influencia se aferra al ideario de masculinidad, de "macho cabrío"(18). Sin embargo, la experimentación y la vivencia de aspectos que naturalizan el autocuidado, no como espectador, sino como participante directo, propició en estos hombres una nueva mirada de los significados atribuidos al autocuidado y la masculinidad ${ }^{(20)}$.

El modelo de masculinidad puede ser diferente al impuesto: solitario, reservado y superficial a lo largo de la vida ${ }^{(21)}$. El hombre puede ser más sensible y no comprometer su razón corporal de ser hombre en la búsqueda de la virilidad ${ }^{(18,21)}$. La resignificación de la masculinidad que se evidencia en este estudio fue acompañada de un movimiento de mirarse a sí mismo, cambiando la relación de ese hombre con la propia salud. Culturalmente, el modelo hegemónico de masculinidad lleva a los hombres tanto a sublimar cuestiones relativas al autocuidado como a ser más vulnerables a los eventos de riesgo de muerte precoz por dolencias evitables, lo que lo torna un ser humano con miedo a enfermarse ${ }^{(22)}$.

Para Gomes ${ }^{(23)}$, esos miedos no pueden ser tomados simplemente como "ignorancia", son reacciones al hecho de que la imagen de masculinidad hegemónica se está rasgando y necesita ser discutida para su deconstrucción. El examen del tacto, por ejemplo, puede ser visto por el hombre como una violación de su masculinidad, si ciertas partes del su cuerpo son intocables. Vergüenza, dolor al ser penetrado, la erección como respuesta fisiológica viola y hiere el modelo hegemónico de masculinidad. En ese contexto, el tacto rectal no involucra solo a la próstata, sino también a la propia masculinidad ${ }^{(24)}$.

En ese contexto, la resignificación de la masculinidad de los participantes se ve acompañada por el quiebre del mito de la invulnerabilidad masculina. En Brasil, ese mito es legitimado por el silenciamiento en relación con los cuidados en salud del hombre en el ámbito del Sistema Único de Salud (SUS), sobre todo en atención primaria ${ }^{(25)}$. Es importante reconocer que la PNAISH hizo emerger nuevas posibilidades para la inserción del hombre en el espacio de la salud pública, a pesar de tener un discurso aún silencioso en cuanto a la subjetividad masculina, que ni siquiera retoma cuestiones relevantes como salud mental y sexualidad ${ }^{(4,17)}$.

De este modo, tales datos contribuyen a un mayor entendimiento de cómo el autoconcepto influye en el autocuidado de la 
salud, pues la concepción acerca del modelo hegemónico de masculinidad, en el que ciertos atributos característicos de esos agentes sociales favorecían una percepción de invulnerabilidad del cuerpo masculino, que genera comportamientos que predisponen a enfermedades y muertes, ha sido deconstruido y se ha gestado un nuevo hombre.

El presente estudio contiene ciertas limitaciones. Al tratarse de una temática poco explorada, se plantean dificultades para abordarla, dado que el propio género masculino aún desconoce derechos y políticas públicas para su salud y conoce poco sobre prevención en salud física y mental. Culturalmente, el hombre inserto en el contexto salud como usuario está relacionado fuertemente con el tabú y el estigma de debilidad y no de opción del cuidado.

Sin embargo, a pesar de estar insertos en problemas más complejos, en situaciones de vulnerabilidad social, estos hombres quebraron paradigmas culturales al insertarse en el grupo de educación y salud y pudieron cambiar perspectivas de su comportamiento, lo que puede culminar en un cambio incluso cultural, que se aparte de la visión del hombre inquebrantable, que nada lo enferma, hacia un buen ser humano.

En el "círculo de cultura" con los profesionales que trabajaban en la unidad de atención primaria, se presentaron la metodología y los resultados de la intervención a partir de los relatos de los participantes. La interacción institucional, intersectorial y multiprofesional fue enriquecedora y proporcionó un vasto encuentro de saberes y servicios interrelacionados, puestos a disposición de la población en cada nueva etapa de la intervención. La integración con otras áreas de salud, como el centro de especialidades odontológicas, los laboratorios municipales y el servicio ambulatorio de lepra, parte del principio de la descentralización de la atención, en el que complementar los servicios es fundamental para enriquecer la integralidad de la atención de los hombres.

Se decidió continuar con las reuniones del grupo de hombres, en horario nocturno, y con periodicidad mensual. Cabe desatacar que esta decisión se consolidó como una estrategia valiosa para el fortalecimiento del vínculo hombres-servicio de salud dado que, el horario de funcionamiento diurno se configura como una barrera institucional para la atención de este público. Tal acción contribuyó, por lo tanto, a aproximarse a lo masculino, desarrollar el sentimiento de pertenencia a la unidad de atención primaria, e incentivar y mejorar el desarrollo de cuidados y prácticas de prevención y promoción de la salud de esos sujetos.

Entre las limitaciones del estudio, no fue posible acompañar a los sujetos en sus búsquedas de cuidados en los servicios de atención primaria luego de la intervención. Esos datos podrían contribuir al entendimiento de la periodicidad de la participación masculina en los espacios de promoción de la salud y la búsqueda de atención. Como punto fuerte, es posible destacar el entendimiento por parte de los profesionales y de los usuarios de salud de que, para la atención de la población masculina, es necesario que los propios profesionales se capaciten, problematicen la realidad del hombre en el proceso saludenfermedad $y$, juntamente con los gestores de salud, vislumbren y operacionalicen estrategias inclusivas para el público masculino, entre ellas, horarios alternativos e implementación de programas específicos.

De acuerdo con Gomes y Nascimento(10), en lo que respecta a la atención de la salud de los hombres $y$, principalmente, para que las acciones dirigidas a este público tengan éxito, resulta necesario comprender la existencia de una pluralidad de formas de ejercer la masculinidad, sin restringirla a un único modelo hegemónico. Esas diversas formas de masculinidad están influenciadas por varios factores, como la raza, clase social, grupo etario, y deben ser considerados por los servicios y profesionales de la salud al elaborar acciones dirigidas a este público.

Si bien al analizar los discursos de los participantes sobre sus definiciones de masculinidad se identificaron aspectos en común, la experiencia posibilitó reflexionar sobre la importancia del desarrollo de acciones estratégicas con la intención de promover la 
aproximación del público masculino al servicio de salud, como el protagonismo y el autocuidado, especialmente, en el marco de la atención primaria, espacio significativo para la prevención de dolencias y la promoción de la salud. Sostener encuentros, aunque sean mensuales, se muestra como un gran avance para la inclusión de los hombres al servicio.

Existe una necesidad de sensibilizar al segmento poblacional en cuestión para la utilización de la atención primaria como puerta de entrada en el Sistema Único de Salud (SUS). En este sentido, resignificar acciones que involucren la salud del hombre y el cambio de actitud profesional para atender a esta población pueden generar en el público masculino un sentido de pertenencia al espacio de promoción, protección y recuperación de la salud.

Ante la actual crisis de las masculinidades y del conflicto identitario relacionado, los resultados evidencian una posibilidad de resignificación de la identidad masculina a partir de la reconstrucción del "ser hombre". Ese proceso demanda enfocarse en el hombre, no como ser indestructible, sino como sujeto que trabaja sus vulnerabilidades. De este modo, el estudio evidencia la participación del hombre en el propio proceso salud-enfermedad bajo la óptica de esa reconstrucción identitaria. Una escasa participación del hombre en su salud trae aparejado el riesgo de reforzar el estereotipo jerárquico que visualiza a los hombres en situación de vulnerabilidad como seres frágiles e indignos de los atributos de fuerza y virilidad.

Aunque las definiciones construidas por estos participantes no se hayan hecho de forma consciente, pueden orientar el modo en que los profesionales de salud orienten futuras acciones en salud dirigidas al público masculino. Resulta necesario, por lo tanto, la realización de más estudios sobre la atención destinada al público masculino, poniendo especial interés en los diversos discursos que circulan en los contextos de salud.

\section{REFERENCIAS BIBLIOGRÁFICAS}

1. Moura EC, Santos W, Neves ACM, Gomes R, Schwatz $\mathrm{E}$. Atenção à saúde dos homens no âmbito da Estratégia Saúde da Família. Ciência \& Saúde Coletiva. 2014;19(2):429-438.

2. Levorato CD, Mello LM, Silva AS, Nunes AA. Fatores associados à procura por serviços de saúde numa perspectiva relacional de gênero. Ciência \& Saúde Coletiva. 2014;19(4):1263-1274.

3. Oliveira MM, Daher DV, Silva JLL, Andrade SSCA. A saúde do homem em questão: busca por atendimento na atenção básica de saúde. Ciência \& Saúde Coletiva. 2015;20(1):273-278.

4. Brasil, Ministério da Saúde. Política nacional de atenção integral a saúde do homem. Brasília: Ministério da Saúde; 2008.

5. Gomes R, Moreira MCN, Nascimento EF, Rebello LEFS, Couto MT, Schraiber LB. Os homens não vêm!: Ausência e/ou invisibilidade masculina na atenção primária. Ciência \& Saúde Coletiva. 2011;16(Sup. 1):S983-S992.
6. Moreira MCN, Gomes R, Ribeiro CR. E agora o homem vem?! Estratégias de atenção à saúde dos homens. Cadernos de Saúde Pública. 2016;32(4): e00060015.

7. Almeida MV. Senhores de si: uma interpretação antropológica da masculinidade. 2a ed. Lisboa: Fim de Século; 2000.

8. Santos HB. Um homem para chamar de seu: uma perspectiva genealógica da emergência da Política Nacional de Atenção Integral à Saúde do Homem. [Dissertação]. Porto Alegre: Universidade Federal do Rio Grande do Sul; 2013.

9. Alves VS. Um modelo de educação em saúde para o Programa Saúde da Família: pela integralidade da atenção e reorientação do modelo assistencial. Interface - Comunicação, Saúde, Educação. 2005;9(16):39-52.

10. Gomes R, Nascimento EF. A produção do conhecimento da saúde pública sobre a relação homem-saúde: uma revisão bibliográfica. Cadernos de Saúde Pública. 2010;22(5):901-911. 
11. Monteiro EMLM, Vieira NFC. Educação em saúde a partir de círculos de cultura. Revista Brasileira de Enfermagem. 2010;63(3):397-403.

12. Bardin L. Análise de conteúdo. Lisboa: Edições 70; 1977.

13. Gomes R, Albernaz L, Ribeiro CRS, Moreira MCN, Nascimento M. Linhas de cuidados masculinos voltados para a saúde sexual, a reprodução e a paternidade. Ciência \& Saúde Coletiva. 2016;21(5):1545-1552.

14. Couto MT, Gomes R. Homens, saúde e políticas públicas: a equidade de gênero em questão. Ciência \& Saúde Coletiva. 2012;17(10):2569-2578.

15. Bento B. Masculinidades críticas e a proposição analítica e relacional nas contemporâneas discussões de gênero. Revista Estudos Feministas. 2015;23(3):1026-1029.

16. Viana Júnior MM. Masculinidades: ampliando o debate. Revista Forum Identidades. 2017;23:87108.

17. Nader MB, Caminoti JM. Gênero e poder: a construção da masculinidade e o exercício do poder masculino na esfera doméstica. Anais do $16^{\circ}$ Encontro Regional de História da ANPUHRIO: Saberes e Práticas Científicas. Rio de Janeiro, Brasil. ANPUH-Rio; 2014.

18. Brilhante AVM, Silva JG, Vieira LJES, Barros NF, Catrib AMF. Construcción del estereotipo del "macho nordestino" brasileño en las letras de forró. Interface - Comunicação, Saúde, Educação. 2018;22(64):13-28.

19. Passos E, Campos GWS. A PNH como um modo de fazer: desafios para a humanização do SUS: Diretrizes para reorganização do trabalho em saúde. Brasília: Ministério da Saúde; 2009.

20. Couto MT, Dantas SMV. Gênero, masculinidades e saúde em revista: a produção da área na revista Saúde e Sociedade. Saúde e Sociedade. 2016;25(4):857-868.

21. Bourdieu P. A dominação masculina. 11a ed. Rio de Janeiro: Bertrand Brasil; 2012.

22. Kimmel MS. A produção simultânea de masculinidades hegemônicas e subalternas. Horizontes Antropológicos. 1998;4(9):103-117.

23. Gomes R, Nascimento EF, Rebello LEFS, Araújo FC. As arranhaduras da masculinidade: uma discussão sobre o toque retal como medida de prevenção do câncer prostático. Ciência \& Saúde Coletiva. 2017;13(6):1975-1984.

24. Medrado B, Lyra J, Azevedo M. "Eu não sou uma próstata, sou um homem!" En: Gomes R, (ed.). Saúde do homem em debate. Rio de Janeiro: Fiocruz; 2011. p. 39-74.

25. Bursztyn I. Estratégias de mudança na atenção básica: avaliação da implantação piloto do Projeto Homens Jovens e Saúde no Rio de Janeiro, Brasil. Cadernos de Saúde Pública. 2008;24(10):22272238.

\section{FORMA DE CITAR}

Paiva Neto FT, Sandreschi PF, Dias MSA, Loch MR. Dificultades del autocuidado masculino: discursos de hombres participantes en un grupo de educación para la salud. Salud Colectiva. 2020;16:e2250. doi: 10.18294/sc.2020.2250.

Recibido: 29-03-2019 | Versión final: 04-12-2019 | Aprobado: 16-12-2019 | Publicado en línea: 06-02-2020

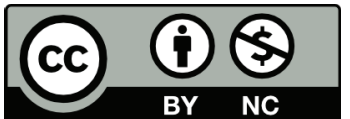

Esta obra está bajo una licencia de Creative Commons Reconocimiento-NoComercial 4.0 Internacional. Reconocimiento - Permite copiar, distribuir y comunicar públicamente la obra. A cambio, se debe reconocer y citar al autor original. No Comercial - Esta obra no puede ser utilizada con finalidades comerciales, a menos que se obtenga el permiso. 